\title{
Theoretical Assessment of Nd:YAG Ceramic Laser Performance by Microstructural and Optical Modeling
}

\author{
Ulrich Aschauer $^{\dagger, \ddagger}$ and Paul Bowen \\ Laboratoire de technologie des poudres (LTP), Ecole polytechnique fédérale de Lausanne (EPFL),
}

1015 Lausanne, Switzerland

\begin{abstract}
An approach combining microstructural and optical modeling based on atomistic simulation results is proposed, in order to assess the performance of $\mathrm{Nd}$ :YAG laser ceramics resulting from luminescence and transparency changes as a function of grain size and dopant concentration. It is found that while small grain sizes enhance transparency, luminescence is reduced, resulting in a trade-off to achieve optimum laser performance. These effects are linked to ceramic production by considering different sintering routes. This improved insight into the microstructural effects of Nd:YAG ceramics on laser performance will allow a more targeted experimental optimization of this new class of laser materials.
\end{abstract}

\section{Introduction}

$I^{N}$ $\mathrm{N}$ recent years, much research ${ }^{1-8}$ has been dedicated to replace the single crystal usually found in Nd:YAG lasers by a ceramic made of the same material. The ceramic would have the advantage to be cheaper to produce and to accommodate higher Nd concentrations ${ }^{8}$ than those possible in single crystals. ${ }^{7,9}$ Surprisingly, although grain boundaries and other defects, are present ceramics usually exhibit a better optical homogeneity than single crystals, ${ }^{10}$ as important crystal structure defects exist in single crystals grown from the melt. ${ }^{8}$ The main challenge in the production of a Nd:YAG laser ceramic is to obtain similar or even better laser performance than conventional single-crystal lasers. Therefore, many advanced ceramic processing techniques have been explored to produce laser ceramics, ${ }^{1,8}$ leading to promising results. Recent attempts ${ }^{1-13}$ have also been made at using nano-sized ceramics, which promise a higher transparency than ceramics with a larger grain size. These small-grained ceramics with a given grain size and dopant composition are, however, extremely difficult to produce and it is as of yet unknown if they will have the desired properties. Therefore, a theoretical assessment of the laser performance as a function of grain size and dopant concentration would be highly desirable in order to optimize the ceramic composition and microstructure in a more targeted, economic way, ultimately leading to better $\mathrm{Nd}$ :YAG laser ceramics.

The present work develops a new microstructural model based on atomistic simulation data previously published, ${ }^{14,15}$ which will be used together with a modified version of an existing optical model for ceramics. ${ }^{16}$ Laser performance strongly depends on transparency and luminescence of the material used. The models will be used to predict these properties of a $\mathrm{Nd}$ :YAG laser ceramic as a function of both the grain size

L.-Q. Chen-contributing editor

Manuscript No. 26330. Received June 11, 2009; approved October 1, 2009

This work was supported by the European Sixth Framework Integrated Project Nanoker No. NMP3-CT-2005-515784.

Author to whom correspondence should be addressed. e-mail: uli.aschauer $@$ epfl.ch

${ }^{\ddagger}$ Present address: Department of Chemistry, Princeton University, Princeton, NJ 08544 and dopant concentration. Moreover, the model will allow insights into the optimal synthesis procedure for these ceramics.

\section{Models}

\section{(1) Microstructural Model}

The requirement for the microstructural model is to give a description of the surface/interface area and the particle/grain volume for a given particle/grain size. The total surface/interface area is, as described in the following, segmented into contributions associated with the different calculated crystallographic surfaces or interfaces, which allows the calculation of segregation properties within a particle or microstructure.

For a powder particle, the classic Wulff construction ${ }^{17}$ is a logical choice for the segmentation as it describes the equilibrium morphology of the particle, and thus its volume and surface area for a given particle size. In this model, we assume the particle size to be the diameter of the smallest sphere containing the Wulff morphology. Wulff morphologies have been calculated using the GEM module within the METADISE ${ }^{18}$ code.

A model microstructure is best described by an arrangement of space filling polyhedrons. For the present study, a space-filling rhombic dodecahedron has been chosen, the volume and surface area of which are given by

$$
\begin{aligned}
& V=\frac{16}{9} \sqrt{3} a^{3} \\
& A=8 \sqrt{2} a^{2}
\end{aligned}
$$

In these equations, $a$ is the side length of one of the rhombic elements of the dodecahedron. The grain size is again taken as the diameter of the smallest enclosing sphere, being $2 a$.

The results of an atomistic simulation study previously published by our group ${ }^{14,15}$ give surface energies for multiple terminations of the morphology dominating surfaces of undoped YAG as well as Nd-doped YAG with segregated dopant ions at the surfaces. Terminations signify surfaces cleaved along the same direction but at different depths in the crystal unit cell. In equilibrium, only the lowest energy termination will appear. This is the case for the Wulff construction, which describes the equilibrium morphology of a powder particle. In a nonequilibrium situation, however, higher energy terminations may also appear. The data given by the atomistic simulations can be used in this sense to approximate a nonequilibrium powder particle, assuming that every termination along every direction contributes to the total surface area of the particle proportional to the inverse of its surface energy as given by

$$
A_{i}=A \frac{\gamma_{i}^{-1}}{\sum_{j} \gamma_{j}^{-1}}
$$

This extension of the Wulff construction gives, under the hypothesis that the selection of calculated surfaces is representative, a good approximation of a nonequilibrium situation. It should be noted that in the present model, no assumption is 
made on how the surfaces are arranged. One can, however, imagine a situation such as surface faceting, where a certain macroscopic surface plane is made up by smaller facets of less energetic surfaces.

The population of interfaces in a microstructure can be described in exactly the same way. The interface area of the rhombic dodecahedron can be assumed to be made up of either only the lowest energy terminations of each type of grain boundary or of all of them, for both situations again in proportion of their inverse interfacial energy as given by Eq. (3).

Based on the volume and surface/interface information provided by the above models, Eq. (4) allows us to calculate the number of yttrium ions per particle/grain $N_{\mathrm{Y}}$, by knowing the number of yttrium ions in the crystallographic unit cell $N_{\mathrm{Y}, \mathrm{UC}}=24$ and its volume $V_{\mathrm{UC}}=1.697 \mathrm{~nm}^{3}$ :

$$
N_{\mathrm{Y}}=V \frac{N_{\mathrm{Y}, \mathrm{UC}}}{V_{\mathrm{UC}}}
$$

The number of neodymium ions per particle/grain $N_{\mathrm{Nd}}$ is linked to $N_{\mathrm{Y}}$ by the nominal bulk dopant concentration $x_{\mathrm{b}, \text { nom }}$ as given by

$$
N_{\mathrm{Nd}}=x_{\mathrm{b}, \mathrm{nom}} N_{\mathrm{Y}}
$$

From the atomistic simulations, ${ }^{14,15}$ the interface concentration of neodymium ions $c_{\mathrm{Nd}, i}$ (ions $/ \mathrm{nm}^{2}$ ) is known, which allows the total number of dopant ions segregated at surfaces/interfaces $N_{\mathrm{Nd}, \text { seg }}$ to be calculated, by knowing the respective surface areas of all crystallographic terminations:

$$
N_{\mathrm{Nd}, \mathrm{seg}}=\sum_{i} A_{i} c_{\mathrm{Nd}, i}
$$

The sum is calculated over the surfaces/interfaces, which appear as given by the equilibrium and nonequilibrium models considered above.

Based on these quantities, it is possible to calculate the fraction of segregated $\mathrm{Nd}$ ions $x_{\mathrm{seg}}$, the effective bulk dopant concentration $x_{\mathrm{b}, \text { eff }}$, and the depletion of the bulk $x_{\mathrm{depl}}$ as given by equations

$$
\begin{aligned}
& x_{\mathrm{seg}}=\frac{N_{\mathrm{Nd}, \mathrm{seg}}}{N_{\mathrm{Nd}}} \\
& x_{\mathrm{b}, \mathrm{eff}}=\frac{N_{\mathrm{Nd}}-N_{\mathrm{Nd}, \mathrm{seg}}}{N_{\mathrm{Y}}} \\
& x_{\mathrm{depl}}=x_{\mathrm{b}, \mathrm{nom}}-x_{\mathrm{b}, \mathrm{eff}}
\end{aligned}
$$

\section{(2) Optical Model}

A model describing the real inline transmittance (RIT) of birefringent ceramic materials has been established by Apetz and van Bruggen. ${ }^{16}$ This model can be adapted to describe the case of a nonbirefringent ceramic having a local variation of the refractive index due to dopant segregation in a region close to the grain boundary. The basic equation describing the RIT (Eq. (10)) is the same as in the original paper by Apetz and van Bruggen:

$$
\mathrm{RIT}=\left(1-R_{\mathrm{s}}\right) \exp (-\mu d)
$$

The thickness of the sample is given by $d, \mu$ is the scattering coefficient of the microstructure considered, and $R_{\mathrm{s}}$ gives the fraction of light reflected at the surface as defined by the perpendicular total Fresnel reflectance:

$$
R_{\mathrm{s}}=\frac{2 R^{\prime}}{1+R^{\prime}}, \quad R^{\prime}=\left(\frac{n-1}{n+1}\right)^{2}
$$

The microstructure is described as spherical grains embedded into a matrix of different refractive index. For the purpose of this paper, the matrix is assumed to have a grain boundary refractive index, whereas the grains all have the refractive index of the pure YAG phase. The volume fraction of the pure YAG phase $x_{\text {pure }}$ is calculated as given by Eq. (12), where $r$ is the grain radius (half the grain size) and $h$ is the thickness of the segregated layer at the grain boundary, as obtained by the atomistic simulations ${ }^{14,15}$ :

$$
x_{\text {pure }}=1-x_{\text {gb }}=\frac{V_{\text {pure }}}{V_{\text {grain }}}=\frac{\frac{4}{3} \pi(r-h)^{3}}{\frac{4}{3} \pi r^{3}}=\left(1-\frac{h}{r}\right)^{3}
$$

The scattering coefficient of such a microstructure is then given by Eq. (13), where $C_{\text {sca }}$ is the scattering cross section of a sphere of radius $r$ and refractive index $n_{\text {grain }}$ embedded in a medium with refractive index $n_{\mathrm{gb}}$, which is calculated using Mie theory ${ }^{19}$ :

$$
\mu=x_{\text {pure }} \frac{1}{V_{\text {grain }}} C_{\text {sca }}=\left(1-\frac{h}{r}\right)^{3} \frac{1}{\frac{4}{3} \pi r^{3}} C_{\text {sca }}
$$

In this model, the volume fraction of the pure phase is larger than the theoretical maximum of $74 \%$ possible for spheres in a hexagonal close-packed array. This is possible because the scattering model considers no overlap of spheres as the scattering is calculated for an isolated sphere. The present approach is, therefore, valid to calculate the scattering in presence of a smaller fraction of grain boundary phase than the fraction of empty space in a hexagonal close-packed array of spheres.

\section{Results and Discussions}

\section{(1) Segregation}

The relevant results of the atomistic simulation studies, ${ }^{15}$ which are used in the present study are summarized in Table I. It can be seen that the calculated surfaces and grain boundaries span a large range of interfacial energies and also show large interfacial areas of up to $3.5 \mathrm{~nm}^{2}$ for the (112) case. The collection of interfaces can, therefore, be considered as a reasonably representative selection of the surfaces and grain boundaries present in real powders and ceramics.

It can be seen from the $c_{\mathrm{Nd}}$ columns that while all surfaces can accommodate $\mathrm{Nd}$-dopant ions to a certain extent, there are grain boundaries that do not accommodate any $\mathrm{Nd}$ ions. ${ }^{15}$ By looking at the grain boundary energies $\gamma$, it can be seen that the boundaries that do not accommodate dopant ions are the ones with a very low energy, and thus has a higher probability of occurring in a microstructure. It should be noted that in these calculations, ${ }^{14,15}$ the spatial extent of the region enriched with dopant ions due to segregation is observed to be of the order of $1 \mathrm{~nm}$, which is significantly smaller than experimental values. ${ }^{20}$ One has to consider, however, the effect of the periodic boundary conditions in conjunction with the relatively small lateral extent of the simulation cells used in these calculations (1-2 nm), which leads to a rapidly increasing effective dopant ion concentration. While in an experiment, lowdopant ion concentrations may still lead to favorable accommodation further from the grain boundaries, these low concentrations are not attainable with today's simulation tools and computers. On the other hand, resolution limitations may result in a broadening of the experimentally measured enriched widths.

In order to evaluate this selective segregation effect within powders and ceramics, the interfacial energies and concentration in Table I have been used in the microstructural model described above. In a first step, the results for powders will be shown and discussed followed by the ones for ceramics.

Figure 1 shows in the top half, the percentage of $\mathrm{Nd}$ ions segregated to surfaces as a function of the powder particle di- 
Table I. Summary of the Previously Published ${ }^{15}$ Atomistic Results Used in this Work

\begin{tabular}{|c|c|c|c|c|c|c|c|}
\hline \multirow[b]{3}{*}{ Plane } & \multirow[b]{3}{*}{ Cut } & \multicolumn{3}{|c|}{ Surface } & \multicolumn{3}{|c|}{ Grain boundary } \\
\hline & & \multicolumn{2}{|c|}{$\gamma\left(\mathrm{J} / \mathrm{m}^{2}\right)$} & \multirow{2}{*}{$\frac{c_{\mathrm{Nd}}\left(\mathrm{nm}^{-2}\right)}{\text { Doped }}$} & \multicolumn{2}{|c|}{$\gamma\left(\mathrm{J} / \mathrm{m}^{2}\right)$} & \multirow{2}{*}{$\frac{c_{\mathrm{Nd}}\left(\mathrm{nm}^{-2}\right)}{\text { Doped }}$} \\
\hline & & Undoped & Doped & & Undoped & Doped & \\
\hline \multirow[t]{5}{*}{$(100)$} & 1 & 2.32 & 2.24 & 2.11 & 2.09 & 1.97 & 4.93 \\
\hline & 2 & 1.81 & 1.77 & 2.82 & 1.93 & 1.73 & 2.82 \\
\hline & 3 & 2.31 & 2.26 & 3.52 & 3.26 & 2.81 & 3.52 \\
\hline & 4 & 2.40 & 1.94 & 2.82 & 2.95 & 2.52 & 5.63 \\
\hline & 5 & 1.91 & 1.86 & 2.11 & 2.84 & 2.82 & 1.41 \\
\hline \multirow[t]{6}{*}{ (110) } & 1 & 2.43 & 2.23 & 2.49 & 1.41 & 1.31 & 1.99 \\
\hline & 2 & 2.36 & 2.33 & 1.00 & 1.79 & 1.61 & 2.99 \\
\hline & 3 & 2.75 & 2.25 & 2.49 & 0.82 & 0.82 & 0.00 \\
\hline & 4 & 2.61 & 2.49 & 1.00 & 2.52 & 2.52 & 0.00 \\
\hline & 5 & 2.67 & 2.47 & 1.99 & 2.31 & 2.31 & 0.50 \\
\hline & 6 & 3.05 & 2.28 & 1.00 & 1.23 & 1.10 & 0.50 \\
\hline \multirow[t]{7}{*}{ (111) } & 1 & 2.33 & 2.33 & 1.22 & 1.90 & 1.78 & 4.07 \\
\hline & 2 & 2.25 & 2.11 & 3.66 & 1.78 & 1.73 & 1.22 \\
\hline & 3 & 2.92 & 2.59 & 2.03 & 2.38 & 2.28 & 2.44 \\
\hline & 4 & 2.48 & 2.21 & 2.85 & 3.49 & 3.43 & 2.44 \\
\hline & 5 & 2.39 & 2.18 & 1.22 & 3.08 & 2.94 & 4.07 \\
\hline & 6 & 2.76 & 2.68 & 2.03 & 3.36 & 3.32 & 2.03 \\
\hline & 7 & 2.95 & 2.83 & 2.44 & 3.13 & 2.93 & 3.66 \\
\hline \multirow[t]{3}{*}{ (112) } & 1 & 2.36 & 2.22 & 2.58 & 1.65 & 1.65 & 0.00 \\
\hline & 2 & 2.61 & 2.52 & 2.29 & 2.70 & 2.54 & 0.86 \\
\hline & 3 & 2.50 & 2.36 & 2.87 & 2.86 & 2.80 & 0.86 \\
\hline
\end{tabular}

Given are the interface energies $(\gamma)$ in the undoped and doped case as well as the concentration of dopant Ions at the interface $\left(c_{\mathrm{Nd}}\right)$ for the doped surfaces and grain boundaries.

ameter. On the left side (Fig. 1(a)), the equilibrium model based on the Wulff equilibrium morphology is shown, whereas the right side (Fig. 1(b)) gives the results for the nonequilibrium case. In general with a decreasing particle size, an exponentially increasing proportion of dopant ions are found at the particle surfaces. It is interesting to note that in the case of a nominally 1 at. \% doped powder at very small grain sizes of around 100 $\mathrm{nm}$, the proportion of segregated $\mathrm{Nd}$ ions reaches $100 \%$ for both situations. This would mean that no dopant ions are located in the bulk of the powder particle but only at its surface. As the nominal dopant concentration increases to 2,4 , and 8 at. $\%$, the fraction of dopant ions at the surface is reduced and reaches values of maximally $20 \%$ at 8 at. $\%$ nominal $\mathrm{Nd}$ concentration for particle sizes of $100 \mathrm{~nm}$. Comparing the equilibrium and nonequilibrium situation, it can be seen that for all concentrations at a given particle size, the proportion of segregated dopant ions is larger for the equilibrium case than for the nonequilibrium case.

The bottom half of Fig. 1 shows depletion (decrease in bulk concentration) due to segregation effects. A value of $-0.5 \%$ means that the effective bulk dopant concentration is $0.5 \%$ below the nominal one i.e. at 3.5 at. $\%$ for the nominally 4 at. $\%$ doped powder. It can be seen that the depletion is virtually independent of the nominal dopant concentration in the equilibrium case (Fig. 1(c)) except for the 1 at.\% case, where a full depletion is reached at particle sizes smaller than $100 \mathrm{~nm}$. At small particle sizes around $100 \mathrm{~nm}$, the effective bulk concentration of dopant ions can drop by up to 1.5 at. \%. In the nonequilibrium case, one can see a dependence of the depletion on the nominal dopant concentration, as a function of the particle size. The lower the nominal concentration, the higher is the depletion of the bulk. This can be explained by the fact that in the equilibrium case, the interfacial concentration of the lowest energy surface terminations occurring is generally very high with values around $2.5-3.5 \mathrm{~nm}^{-2}$. In the nonequilibrium case also, surfaces with only $1 \mathrm{~nm}^{-2}$ appear, which leads overall to a smaller capacity of the powder surface to accommodate dopant ions. This means that at higher nominal doping concentrations, saturation of the surfaces will be attained and more dopant ions remain in the bulk. It has to be noted that the present model does not take into account the formation of second phase precipitates, which may occur at high surface dopant concentrations.

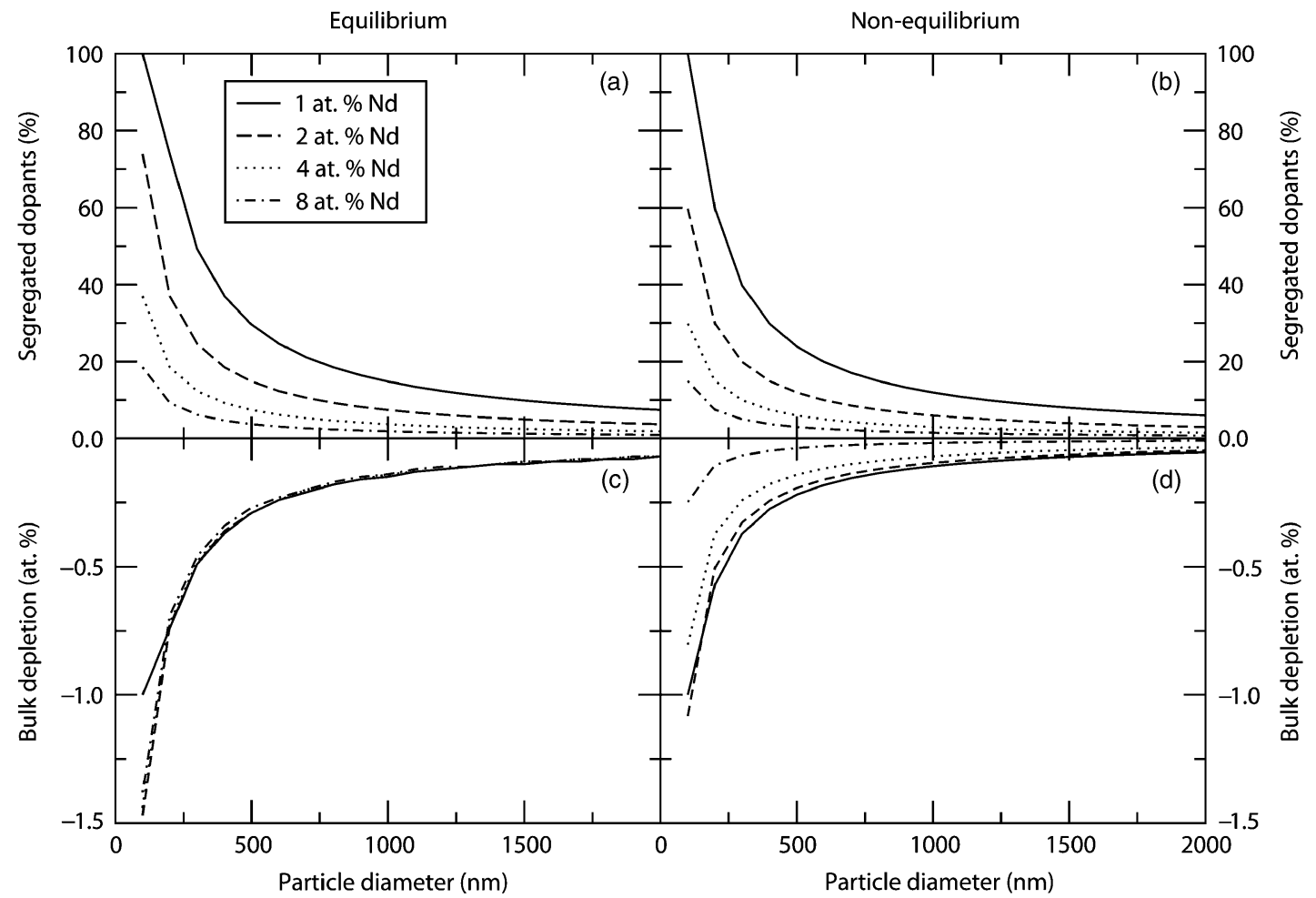

Fig. 1. The fraction of segregated dopant ions at 1,2, 4, and 8 at.\% nominal dopant concentration for (a) the equilibrium powder case and (b) the nonequilibrium powder case. The resulting depletion of the bulk is shown in (c) for the equilibrium case and (d) for the nonequilibrium case. 
In Fig. 2, the results of the calculations for a ceramic microstructure are shown. For ceramics, the same general trends for the dependence on both grain size and nominal doping concentration are observed as for powders. Comparing these results with the ones for powders, it is interesting to note that while for the powder, the fraction of segregated dopant ions is slightly larger in the equilibrium case, the opposite is observed in a ceramic microstructure, where the equilibrium model shows a significantly lower proportion of segregated dopant ions. The origin for this behavior can be found in the fact that two of the low-energy grain boundaries (110) and (112) do not accommodate any dopant ions, which will result in a very low overall dopant accommodation in the equilibrium microstructure. On the other hand, for the nonequilibrium ceramic, dopant segregation proportions are about as high as for the powders. This is also reflected by the depletion of the bulk, where for the equilibrium case only a very small reduction of the nominal dopant concentration is observed, whereas in the nonequilibrium case, the reduction is much larger and also very dependent on the nominal dopant concentration.

\section{(2) Luminescence and Transparency}

So far, the segregation of Nd-dopant ions in YAG powders and ceramics has been described without taking into consideration the effects this segregation may have on the properties of such a material in laser applications. In the following, two possible effects will be outlined and analyzed.

Nd-dopant ions in YAG have to have a very specific crystal environment ${ }^{21}$ in order to present the precise energy levels required for laser emission. It will not be discussed here whether sites with this specific environment can be found close to surfaces or grain boundaries because the following consideration will show that the requirement for such sites is irrelevant. The concentration of dopant ions in a grain boundary as calculated by the atomistic methods within the enriched layer is locally 18 at. \% and more. It is well known that with increasing concentration of the luminescent dopant ions, their luminescence lifetime is decreased, an effect commonly referred to as "concentration quenching." 22 The data in Dong et al. ${ }^{22}$ show that for an increase from 1 to 3 at.\% $\mathrm{Nd}$ concentration, the luminescence lifetime is reduced by half. For much higher local concentrations predicted by atomistic simulations in the enriched layer, a much more severe reduction can be expected rendering the dopant ions inactive with respect to laser emission. This means that one will want to have a minimal concentration of dopant ions at the grain boundary, as these ions have a low luminescence and will not significantly contribute to laser emission. This local concentration quenching effect in the grain boundary was recently seen in an experiment. ${ }^{20}$ With respect to the data shown in Figs. 1 and 2, this would mean that for a powder, the nonequilibrium situation is slightly more favorable, whereas for the ceramic, it is clearly the equilibrium case, which will maximize the effective bulk concentration and thus luminescence. In general, for larger particle and grain sizes, the lower interface volume fraction will lead to less quenched Nd-dopant ions, and therefore a higher luminescence.

From the above discussion, it can be seen that increasing the nominal doping concentration can be a solution to retain a high effective dopant concentration in laser ceramics. The resulting accumulation of dopant ions at the surfaces and grain boundaries can, however, have the effect of slightly changing the refractive index in the enriched layer. These changes can lead to light scattering and deteriorate the high optical transparency required for laser applications, as discussed in. ${ }^{20}$ This effect has been investigated by using the optical model described earlier.

A thickness of the enriched layer at the grain boundary of $0.5 \mathrm{~nm}$ has been considered, which is a good average of the values observed in the atomistic calculations. ${ }^{15}$ In order to get a rough idea of the change in refractive index to be expected, atomistic calculations for a bulk unit cell with an increasing number of $\mathrm{Nd}$ ions substituted for $\mathrm{Y}$ have been performed using the GULP $^{23,24}$ code with Lewis and Catlow ${ }^{25}$ potentials. This potential set includes a core-shell mode ${ }^{26}$ for oxygen and can, therefore, to a certain degree take into account polarization and consequently variations of the dielectric constant and refractive index. Details about the atomistic method can be found elsewhere. ${ }^{14}$ While this is a good first approximation, quantum mechanical methods would certainly provide a more reliable results, however, at a much higher computational cost. This

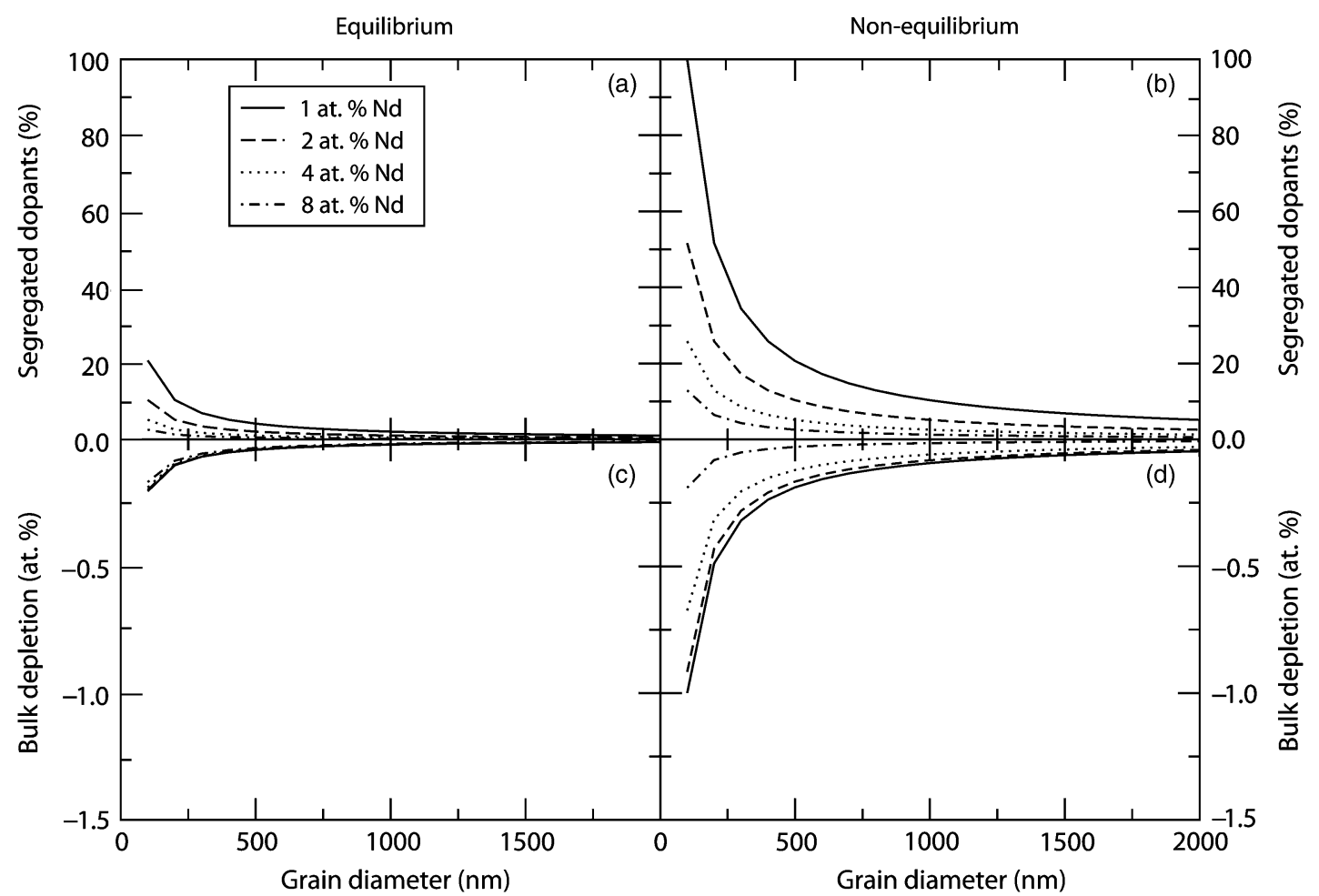

Fig. 2. The fraction of segregated dopant ions at 1,2, 4, and 8 at. \% doping rate for (a) the equilibrium ceramic case and (b) the nonequilibrium ceramic case. The resulting depletion of the bulk is shown in (c) for the equilibrium case and (d) for the nonequilibrium case. 
cost may not be worth paying because even if the absolute magnitude of the refractive indices calculated might have a low accuracy, the optical model only depends on the relative difference in refractive indices. Figure 3 shows that the evolution of the refractive index with increasing $\mathrm{Nd}$ concentration follows a decreasing linear trend. Average interface dopant fractions found in the enriched layers calculated are $0.11-0.26$ for the equilibrium and nonequilibrium case. ${ }^{15}$ This means that the grain boundary will have a deviation in the refractive index between -0.0035 and -0.0069 . These values are slightly higher than those found in previous discussions. ${ }^{20}$ In the following calculations, a grain refractive index of 1.597 and grain boundary refractive indices ranging from 1.590 to 1.597 (pure YAG) have been used to evaluate the effect of the degree of segregation on the transparency.

For laser applications, it is interesting to calculate the RIT for a typical wavelength for the laser emission $(1065 \mathrm{~nm})$ as well as in the visible range $(640 \mathrm{~nm})$. Figure 4 shows the evolution of the RIT as a function of grain size for these two wavelengths considering a sample thickness of $0.8 \mathrm{~mm}$. It can be seen that the reduction in the transparency of the material is more severe in the visible range, however, still not negligible at the infrared laser wavelength. For both cases even at a very low refractive index change of 0.001 , a reduction of $20 \%$ and $40 \%$, respectively, is found at large grain sizes of about $10 \mu \mathrm{m}$. As the grain sizes decrease toward into the submicrometer range, the loss in transparency reduces rapidly and should be negligible for nanoceramics below $100 \mathrm{~nm}$ at either wavelength, even for high interfacial dopant concentrations.

The data obtained from the optical model show that for a given interfacial concentration, smaller grain sizes are favorable for best transparency. This is in contrast to the data obtained from the microstructural model, which show that for smaller grains, the laser efficiency is reduced due to quenched luminescent dopant ions at the grain boundaries. For optimal laser performance, both transparency and high luminescence are required. According to the present model, both cannot be maximized simultaneously, which means that an optimal laser performance should be realized at an intermediate grain size, which gives the best trade-off between transparency and luminescence.

\section{(3) Ceramic Production Route}

In the preceding paragraphs, the state of the initial powder and the final sintered ceramic has been discussed with respect to luminescence and transparency, without considering how the powder and ceramic are produced. The terms equilibrium and nonequilibrium have so far been used only to characterize the population of interfaces found on a powder or in a ceramic, without linking them to a certain experimental situation. In an experiment, an equilibrium powder would be at or close to its

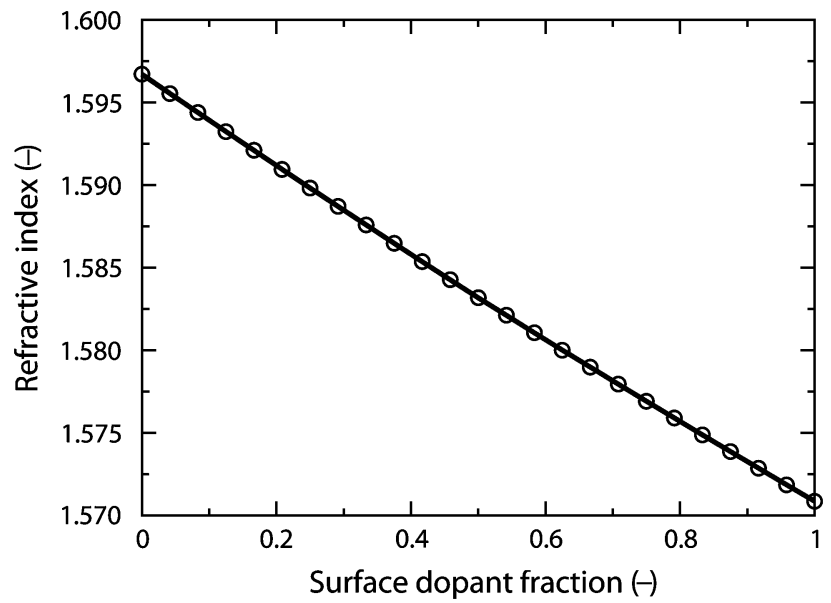

Fig. 3. Evolution of the refractive index as a function the surface fraction in $\mathrm{Nd}$.

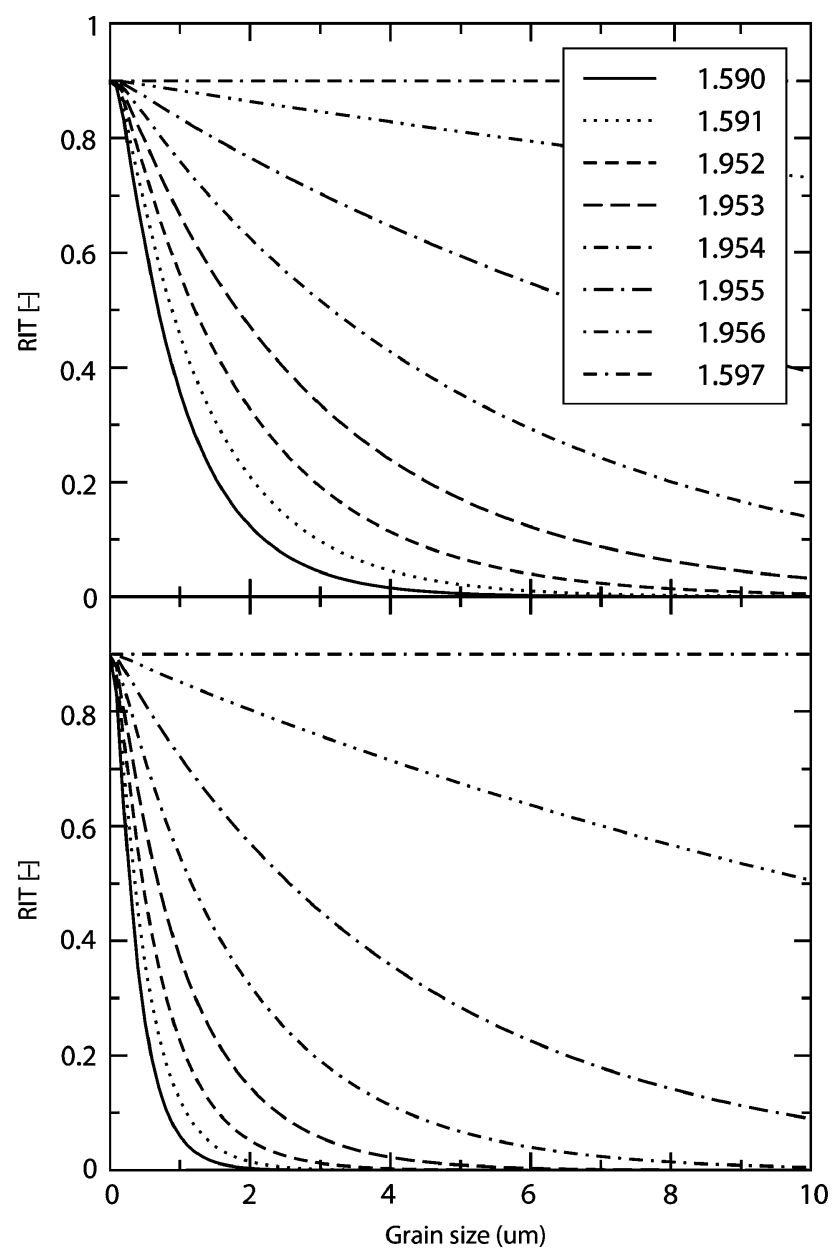

Fig. 4. Real inline transmittance (RIT) as a function of the grain size for (top) a laser wavelength of $1065 \mathrm{~nm}$ and (bottom) a visible wavelength of $640 \mathrm{~nm}$ evaluated for grain boundary refractive indices ranging from 1.590 (25\% interface dopant fraction) to 1.597 (pure YAG).

Wulff morphology, which is usually found for powders precipitated at low growth rates or obtained by a hydrothermal treatment. ${ }^{27}$ A nonequilibrium powder would be formed without giving sufficient time and/or temperature for its morphology to equilibrate such as by a flame method or milling. On the other hand, for dense ceramics the degree of equilibrium will be determined by the time given for the microstructure to evolve. In this respect, a nonequilibrium ceramic is more likely to be produced by a fast sintering method such as spark plasma sintering (SPS), ${ }^{28}$ whereas a conventionally sintered ceramic with an isothermal hold at high temperature ${ }^{29}$ will be closer to equilibrium. One can now analyze the processing routes where one starts from an equilibrium or nonequilibrium powder and sinters it either slowly or quickly. Although simplistic, this approach gives valuable information on the effect a chosen production route will have on the laser performance.

(1) In the case of a nonequilibrium powder that is sintered rapidly, the amount of dopant ions at the interface is about the same in the powder and ceramic state. This means that the dopant ions do not have to migrate in order to be accommodated in the ceramic, greatly reducing the risk of precipitate formation. The presence of a large number of high-energy surfaces in the powder can lead to grain growth, which is, however, limited by the short sintering time. A ceramic produced in this way would be expected to have a moderate size grains and a high number of quenched dopant ions at the grain boundaries. This means that both transparency and luminescence are expected to be low.

(2) When a nonequilibrium powder is sintered slowly, a significantly smaller number of dopant ions can be accommodated 
in the ceramic, compared with the powder. This means that there is a risk of a second phase formation unless the dopant ions can diffuse back to the bulk. Also, the high-energy surfaces combined with the long sintering time can lead to extensive grain growth. The resulting ceramic will, therefore, have a rather coarse microstructure; however, only very few dopant ions are quenched at grain boundaries. This leads to low transparency but high luminescence.

(3) For the situation where an equilibrium powder is sintered slowly, dopant ion relocation is required if second phase precipitation is to be avoided. Grain growth is expected to be moderate even if the sintering time is long as only low-energy surfaces are present. A high number of dopant ions are found in the bulk and active for laser emission. While the transparency is still expected slightly less than optimal, luminescence should be high.

(4) Finally, in the case of an equilibrium powder sintered rapidly, limited dopant diffusion is required and the risk for second phase formation should be low. Grain growth is limited by both low-energy powder surfaces and short sintering times. However, a significant fraction of dopant ions are predicted to be found at the grain boundaries. A very high transparency results, however, at the cost of a small reduction in luminescence.

The situations discussed above present extreme cases, experiments normally being located in between and the magnitude of the effects also depending on the nominal $\mathrm{Nd}$ concentration. It is, however, possible to state that a slower sintering (more equilibrium) will favor luminescence, whereas a more equilibrium powder will lead to increased transparency (less grain growth). An approach with close to equilibrium powders and a rather slow sintering would lead to the best laser properties; however, good results should also be possible with equilibrium powders and fast sintering methods.

Another possible processing route, which is not specifically included in the above, would be to use powders obtained from coprecipitation or other low-temperature methods, having the advantage of inhibiting segregation. However, powders produced by these low-temperature routes rarely form the ceramic phase directly and often require either a heat treatment or a longer sintering cycle for the phase transformation to take place. And although segregation may be less important, it will not be completely inhibited. These powders may, therefore, have a slight advantage in terms of the amount of dopants to be relocated during slow sintering but the results will strongly depend on the degree of intermixing of the primary phases and thus the temperatures and times required for the formation of the YAG phase.

It is interesting to note that experiments ${ }^{13}$ reported a strong concentration dependence of the luminescence for ceramics but not for powders. Their powders produced by the Pechini method should be close to equilibrium, whereas their highpressure-low-temperature-sintered ceramics can be expected not to be in equilibrium. This experimental data, therefore, seem to support the microstructural model predictions, where almost no concentration dependence of the depletion is observed for the equilibrium powder but it is present for the nonequilibrium ceramic. While the proposed model seems to capture certain experimentally observed trends, a complete validation is still impossible as systematic grain-size-dependent data are yet unavailable. The present work should, nevertheless, allow a more fundamental understanding of the performance-affecting effects in Nd:YAG laser ceramics, leading to more focused research and faster progress in the development of this new class of laser materials.

\section{Conclusion}

This work applies two separate models to assess the expected performance of ceramic lasers as a function of microstructural and processing parameters. Microstructural modeling predicts that luminescence and therefore laser power should increase with increasing grain size, whereas optical modeling shows that transparency is higher at lower grain sizes. Therefore, in an experiment a tradeoff between these two effects has to be found to maximize laser performance, which strongly depends on both transparency and luminescence.

It has also been predicted that the processing route can influence both transparency and luminescence, by modifying the microstructure. While a starting powder approach equilibrium may reduce grain growth and therefore increase transparency, longer sintering times would limit segregation effects and increase the number of dopant ions active for laser emission and therefore the luminescence.

The present work opens the path to a new understanding of $\mathrm{Nd}$ :YAG laser ceramics and their production, which will help in the experimental optimization of this new class of materials.

\section{References}

${ }^{1}$ A. Ikesue, K. Kamata, and K. Yoshida, "Synthesis of $\mathrm{Nd}^{3+}, \mathrm{Cr}^{3+}$-Codoped YAG Ceramics for High-Efficiency Solid-State Lasers," J. Am. Ceram. Soc., 78 [9] 2545-7 (1995)

${ }^{2}$ A. Ikesue, K. Kamata, and K. Yoshida, "Effects of Neodymium Concentration on Optical Characteristics of Polycrystalline Nd: YAG Laser Materials,' J. Am. Ceram. Soc., 79 [7] 1921-6 (1996).

${ }^{3}$ J. Lu, M. Prabhu, J. Song, C. Li, J. Xu, K. Ueda, A. A. Kaminskii, H. Yagi, and T. Yanagitani, "Optical Properties and Highly Efficient Laser Oscillation of Nd: YAG Ceramics," App. Phys. B-Lasers Opt., 71 [4] 469-73 (2000).

${ }^{4}$ V. Lupei, A. Lupei, N. Pavel, T. Taira, and A. Ikesue, "Comparative Investigation of Spectroscopic and Laser Emission Characteristics Under Direct 885-nm Pump of Concentrated Nd: YAG Ceramics and Crystals," App. Phys. B-Lasers Opt., 73 [7] 757-62 (2001).

${ }^{5}$ J. R. Lu, K. Ueda, H. Yagi, T. Yanagitani, Y. Akiyama, and A. A. Kaminskii, "Neodymium Doped Yttrium Aluminum Garnet $\left(\mathrm{Y}_{3} \mathrm{Al}_{5} \mathrm{O}_{12}\right)$ Nanocrystalline Ceramics - A New Generation of Solid State Laser and Optical Materials," J. Alloys Comp., 341 [1-2] 220-5 (2002).

${ }^{6}$ J. Lu, H. Yagi, K. Takaichi, T. Uematsu, J. F. Bisson, Y. Feng, A. Shirakawa, K. I. Ueda, T. Yanagitani, and A. A. Kaminskii, " $110 \mathrm{~W}$ Ceramic $\mathrm{Nd}^{3+}$ : $\mathrm{Y}_{3} \mathrm{Al}_{5} \mathrm{O}_{12}$ Laser," App. Phys. B-Lasers Opt., 79 [1] 25-8 (2004).

${ }^{7}$ N. Pavel, V. Lupei, J. Saikawa, T. Taira, and H. Kan, "Neodymium Concentration Dependence of $0.94-, 1.06-$ and 1.34-mm Laser Emission and of Heating Effects Under 809- and 885-nm Diode Laser Pumping of Nd: YAG," Appl. Phys. $B$-Lasers Opt., 82 [4] 599-605 (2006).

${ }^{8}$ A. Ikesue and Y. L. Aung, "Synthesis and Performance of Advanced Ceramic Lasers," J. Am. Ceram. Soc., 89 [6] 1936-44 (2006).

${ }^{9}$ J. A. L'huillier, G. Bitz, V. Wesemann, P.von Loewis, R. Wallenstein, A. Borsutzky, L. Ackermann, K. Dupre, D. Rytz, and S. Vernay, "Characterization and Laser Performance of a New Material: 2 at.\% Nd:YAG Grown by the Czochralski Method," Appl. Opt., 41 [21] 4377-84 (2002).

${ }^{10}$ A. Ikesue and K. Yoshida, "Scattering in Polycrystalline Nd: YAG Lasers," J. Am. Ceram. Soc., 81 [8] 2194-6 (1998).

${ }^{11}$ D. Hreniak, R. Fedyk, A. Bednarkiewicz, W. Strek, and W. Lojkowski, "Luminescence Properties of Nd: YAG Nanoceramics Prepared by Low Temperature High Pressure Sintering Method," Opt. Mater., 29 [10] 1244-51 (2007).

${ }^{12}$ W. Strek, A. Bednarkiewcz, D. Hreniak, P. Mazur, and W. Lojkowski, "Fabrication and Optical Properties of Transparent $\mathrm{Nd}^{3+}$ : YAG Nanoceramics," J. Lumin., 122, 70-3 (2007)

${ }^{13}$ D. Hreniak, W. Strek, P. Gluchowski, R. Fedyk, and W. Lojkowski, "The Concentration Dependence of Luminescence of Nd: $\mathrm{Y}_{3} \mathrm{Al}_{5} \mathrm{O}_{12}$ Nanoceramics," J. Alloys Compd., 451 [1-2] 549-52 (2008).

${ }^{14}$ U. Aschauer, P. Bowen, and S. C. Parker, "Atomistic Modeling Study of Surface Segregation in Nd: YAG,” J. Am. Ceram. Soc., 89 [12] 3812-6 (2006)

${ }^{15}$ U. Aschauer, P. Bowen, and S. C. Parker, "Surface and Mirror Twin Grain Boundary Segregation in Nd: YAG: An Atomistic Simulation Study," J. Am. Ceram. Soc., 91 [8] 2698-705 (2008).

${ }^{16}$ R. Apetz and M. P. B. van Bruggen, "Transparent Alumina: A LightScattering Model," J. Am. Ceram. Soc., 86 [3] 480-6 (2003).

${ }^{17}$ G. Wulff, "On the Question of Speed of Growth and Dissolution of Crystal Surfaces," Z. Krystallogr. Mineral., 34 [5/6] 449-530 (1901).

${ }^{18}$ G. W. Watson, E. T. Kelsey, N. H. deLeeuw, D. J. Harris, and S. C. Parker, "Atomistic Simulation of Dislocations, Surfaces and Interfaces in MgO," J. Chem. Soc.-Faraday Trans., 92 [3] 433-8 (1996).

${ }^{19} \mathrm{G}$. Mie, "Articles on the Optical Characteristics of Turbid tubes, Especially Colloidal Metal Solutions," Ann. Phys., 25 [3] 377-445 (1908).

${ }^{20}$ M. O. Ramirez, J. Wisdom, H. Li, Y. L. Aung, J. Stitt, G. L. Messing, V. Dierolf, Z. Liu, A. Ikesue, R. L. Byer, and V. Gopalan, "Three-Dimensional Grain Boundary Spectroscopy in Transparent High Power Ceramic Laser Materials," Opt. Express, 16 [9] 5965-73 (2008).

${ }^{21}$ G. A. Kumar, J. R. Lu, A. A. Kaminskii, K. I. Ueda, H. Yagi, T. Yanagitani, and N. V. Unnikrishnan, "Spectroscopic and Stimulated Emission Characteristics of $\mathrm{Nd}^{3+}$ in Transparent YAG Ceramics," IEEE J. Quantum Electron., 40 [6] 74758 (2004).

${ }^{22}$ J. Dong, A. Rapaport, M. Bass, F. Szipocs, and K. Ueda, "TemperatureDependent Stimulated Emission Cross Section and Concentration Quenching in Highly Doped $\mathrm{Nd}^{3+}$ : YAG Crystals," Phys. Status Solidi A-Appl. Mater. Sci., 202 [13] 2565-73 (2005). 
${ }^{23}$ J. D. Gale, "GULP: A Computer Program for the Symmetry-Adapted Simulation of Solids," J. Chem. Soc. - Faraday Trans., 93 [4] 629-37 (1997).

${ }^{24}$ J. D. Gale and A. L. Rohl, "The General Utility Lattice Program (GULP)," Mol. Simul., 29 [5] 291-341 (2003).

${ }^{25}$ G. V. Lewis and C. R. A. Catlow, "Potential Models for Ionic Oxides," J. Phys. C-Solid State Phys., 18 [6] 1149-61 (1985).

${ }^{26}$ B. G. Dick and A. W. Overhauser, "Theory of the Dielectric Constants of Alkali Halide Crystals," Phys. Rev., 112 [1] 90-103 (1958).
${ }^{27}$ U. Aschauer, F. Jones, W. Richmond, P. Bowen, A. L. Rohl, G. M. Parkinson, and H. Hofman, "Growth Modification of Hematite by Phosphonate Additives," J. Cryst. Growth, 310 [3] 688-98 (2008).

${ }^{28}$ Z. Zhao, V. Buscaglia, P. Bowen, and M. Nygren, "Spark Plasma Sintering of Nano-Crystalline Ceramics," Eur. Ceram., 264-268 [Parts 1-3] 2297-300 (2004).

${ }^{29}$ P. Bowen, C. Carry, D. Luxembourg, and H. Hofmann, "Colloidal Processing and Sintering of Nanosized Transition Aluminas," Powder Technol., 157 [1-3] 100-7 (2005). 